\title{
BMJ Global Health Antimicrobial use and resistance data in human and animal sectors in the Lao PDR: evidence to inform policy
}

\author{
Vilada Chansamouth (1) , 1,2,3 Mayfong Mayxay (1) ,2,3,4 David AB Dance (1) ,2,3,5 \\ Tamalee Roberts (D) ,2,3 Rattanaxay Phetsouvanh, ${ }^{6}$ Bouakham Vannachone, ${ }^{6}$ \\ Manivanh Vongsouvath, ${ }^{1,2}$ Viengmon Davong, ${ }^{1,2}$ Phout Inthavong, ${ }^{7}$ \\ Syseng Khounsy, ${ }^{7}$ Bounxou Keohavong, ${ }^{8}$ Valy Keoluangkhot, ${ }^{9}$ \\ Khamla Choumlivong, ${ }^{10}$ Nicholas PJ Day (i) , ${ }^{3,11}$ Paul Turner (i) , 3,12 \\ Elizabeth A Ashley (D) , 2,3 H. Rogier van Doorn (D) ,", ${ }^{3,13}$ Paul N Newton (D) 2,3,5,11
}

To cite: Chansamouth V, Mayxay M, Dance DAB, et al. Antimicrobial use and resistance data in human and animal sectors in the Lao PDR: evidence to inform policy. BMJ Global Health 2021;6:e007009. doi:10.1136/ bmjgh-2021-007009

Handling editor Seye Abimbola

- Additional supplemental material is published online only. To view, please visit the journal online (http://dx.doi.org/10. 1136/bmjgh-2021-007009)

Received 30 July 2021 Accepted 23 0ctober 2021
Check for updates

(c) Author(s) (or their employer(s)) 2021. Re-use permitted under CC BY. Published by BMJ.

For numbered affiliations see end of article.

Correspondence to Dr Vilada Chansamouth; vilada@tropmedres.ac

\section{ABSTRACT}

Objectives To review the scientific evidence base on antimicrobial use (AMU) and antimicrobial resistance (AMR) in human and animal sectors in the Lao PDR (Laos).

Methods We reviewed all publications from July 1994 (the first article describing AMR in Laos) to December 2020. Electronic searches were conducted using Google Scholar and PubMed with specific terms relating to AMR and AMU in Lao, French and English languages.

Findings We screened 1,357 peer-reviewed and grey reports by title and abstract and then full articles/reports. of 80 included, $66(83 \%)$ related to human health, nine $(11 \%)$ to animal health, four (5\%) to both animal and human health and one (1\%) to the environment. Sixtytwo $(78 \%)$ were on AMR and $18(22 \%)$ on AMU. Extended spectrum beta lactamase-producing Escherichia coli was the greatest concern identified; the proportion of isolates increased fivefold from 2004 to $2016(2 / 28(7 \%)$ to $27 / 78$ (35\%)) from blood cultures submitted to the Microbiology Laboratory, Mahosot Hospital, Vientiane. Carbapenem resistant Escherichia coli was first identified in 2015. Methicillin-resistant Staphylococcus aureus (MRSA) was uncommon, with 15 cases of MRSA from blood cultures between its first identification in 2017 and December 2020. AMR patterns of global antimicrobial resistance surveillance system (GLASS) target pathogens from livestock were less well documented. There were few data on AMU in human health and none on AMU in livestock. The first hospital AMU survey in Laos showed that $70 \%$ $(1,386 / 1,981)$ of in-patients in five hospitals from 2017 to 2018 received antimicrobial(s). Antibiotic self-medication was common.

Conclusion AMR in Laos is occurring at relatively low proportions for some GLASS pathogens, giving the country a window of opportunity to act quickly to implement strategies to protect the population from a worsening situation. Urgent interventions to roll out new guidelines with enhanced one-health antibiotic stewardship, reduce antibiotic use without prescriptions, enhance surveillance and improve understanding of $\mathrm{AMU}$ and AMR are needed.

\section{Key questions}

What is already known?

- Irrational use of antimicrobials driving antimicrobial resistance (AMR) is a critically important issue worldwide

- Data on antimicrobial susceptibility patterns of the global AMR surveillance system pathogens are commonly available in wealthier countries but fewer data are available in low-income and middle-income countries.

- There are scattered reports of AMR and antimicrobial use (AMU) in Laos, but they have not been synthesised to inform policy and implementation.

What are the new findings?

- Extended spectrum beta lactamase-producing Escherichia coli has become the greatest AMR concern in Laos.

- Carbapenem resistant E. coli and Klebsiella pneumo niae have been reported recently. Colistin resistant $E$. coli and K. pneumoniae have been reported in both livestock and a healthy individual.

- Data suggest a high proportion of hospital AMU in human health but no AMU data in animal health were found.

What do the new findings imply?

- The AMR situation in Laos is not as severe as in surrounding countries, suggesting that Lao has the opportunity to act quickly to control and combat AMR to avert a worsening situation.

- There is an urgent need for AMR and AMU monitoring in both hospitals and the community, improved antibiotic stewardship in humans and livestock and enhanced control of antibiotic use without prescriptions.

- More research is required to investigate appropriate solutions for the Lao context.

\section{BACKGROUND}

Antimicrobial resistance (AMR) is of major global public health concern, increasing in frequency for key pathogens and key 
antimicrobials. Evidence suggests that this is accelerated by antimicrobial use (AMU). AMR results in both direct consequences, such as longer duration of illness and hospital stay, and increased morbidity/mortality, and indirect consequences such as reduced productivity caused by sickness and increased family, community and health system economic burden. ${ }^{12}$ Since 2000, the number of antibiotics under development has fallen sharply. ${ }^{3}$ It has been estimated that approximately 700,000 people die every year from resistant pathogens, including bacterial infections, tuberculosis (TB), HIV and malaria. ${ }^{4}$ If AMR continues to increase with no appropriate action being taken, this could contribute 10 million excess deaths by $2050{ }^{4}$

Laos is a low- and middle-income country, bordered by Thailand, Cambodia, Vietnam, China and Myanmar. National action plans on AMR are available in all these countries, and Laos, and most of surrounding countries (except China and Vietnam) participate in the global antimicrobial resistance surveillance system (GLASS). ${ }^{5}$ However, in Laos, as is common elsewhere, different sectors (healthcare, agriculture, drug regulatory authorities) tend to work on their own strategies with little interaction. In October 2016, the Global Antibiotic Resistance Partnership-Laos committee was established as a Ministry of Health $(\mathrm{MoH})$ technical working group to review the scientific evidence base to inform efforts to combat AMR in Laos, and provide evidence to the MoH AMR Surveillance and Control Committee. This report summarises the evidence base on AMU and bacterial resistance patterns in humans, animals and the environment in Laos, to identify important gaps and discusses the implications for health policy.

\section{METHODS}

\section{Information sources and inclusion/exclusion criteria}

This narrative review focuses on the existing evidence on the prevalence of antimicrobial resistance among bacterial pathogens, particularly GLASS target pathogens, Mycobacterium tuberculosis and other available AMR pathogens in humans, animals and the environment in Laos. The review also describes data on AMU in these three sectors. All the peer-reviewed and grey literatures relating to Lao AMU and AMR data in humans, animals and the environment from 1994 (the year of the first published article on AMR in Laos) to 31 December 2020 in Lao, French and English languages were screened by title and abstract. We also included epidemiological studies, case studies and available raw data/reports from various governmental and non-governmental sectors in Laos.

This review does not include peer-reviewed or grey literatures relating to antimalarial, antiprotozoal, antihelmintic, antifungal and antiviral resistance.

\section{Published literature}

Electronic searches were conducted using Google Scholar and PubMed. The search terms used were 'Laos,
Lao, antimicrobial susceptibility, antimicrobial resistance, antimicrobial use, animals, pharmacy, prescription, prevention, self-medication, environment, Escherichia coli, Klebsiella pneumoniae, Acinetobacter baumannii, Staphylococcus aureus, Streptococcus pneumoniae, Salmonella spp, Shigella spp, Neisseria gonorrhoeae, Salmonella enterica serovar Typhi, Salmonella Paratyphi, Vibrio cholerae, Mycobacterium tuberculosis, extended spectrum beta lactamase (ESBL) and methicillin-resistant Staphylococcus aureus (MRSA)'. Eligible articles were included after reading the abstracts. Full articles were then reviewed to obtain relevant data for inclusion.

\section{Grey literature}

Relevant Lao Government departments were visited and data and reports requested. Annual reports, student project theses, results of projects on antibiotic use and resistance in Laos were requested from the Lao Food and Drug Department, Department of Healthcare and Rehabilitation, National Tuberculosis Centre, Microbiology Laboratory of Mahosot Hospital (the largest central hospital in Laos-the majority of laboratory data in this review were from this laboratory, which acts as a reference microbiology laboratory and provides services across the country), Livestock and Fisheries Department of Ministry of Agriculture \& Forestry, Faculty of Agriculture of Lao National University and Institut de la Francophonie pour la Médecine Tropicale (IFMT).

We did not grade the quality of evidence, nor contact the authors for more information. The review results were described without further analysis.

\section{Patient and public involvement}

There was no patient or public involvement in the planning or conduct of this work.

\section{FINDINGS}

From 28 July 1994 to 31 December 2020, 1,357 peerreviewed and grey literature reports were identified and screened by title and abstract; $487(36 \%)$ were peer reviews from electronic searches and 870 (64\%) were datasets or grey literature. After review, 80 peer-reviewed and grey literature reports were included. Of these, 62 $(78 \%)$ were related to AMR and $18(22 \%)$ to AMU in Laos (figures 1 and 2).

Of 80 data sources, $66(83 \%)$ related to human health only, $9(11 \%)$ related to animal health, $4(5 \%)$ covered both human and animal health and one $(1 \%)$ related to environmental AMR pathogens (online supplemental appendices 1-4). Forty-one (51\%) were from, or related to, work done by the Lao-Oxford-Mahosot HospitalWellcome Trust Research Unit (LOMWRU), Mahosot Hospital, Vientiane.

Antimicrobial susceptibility testing (AST) described in this review was based on the clinical and laboratory standard institute (CLSI) methods, as was used in all microbiology laboratories in Laos until recently. It was replaced by the European Committee on AST system in mid-2019. ${ }^{6}$ 


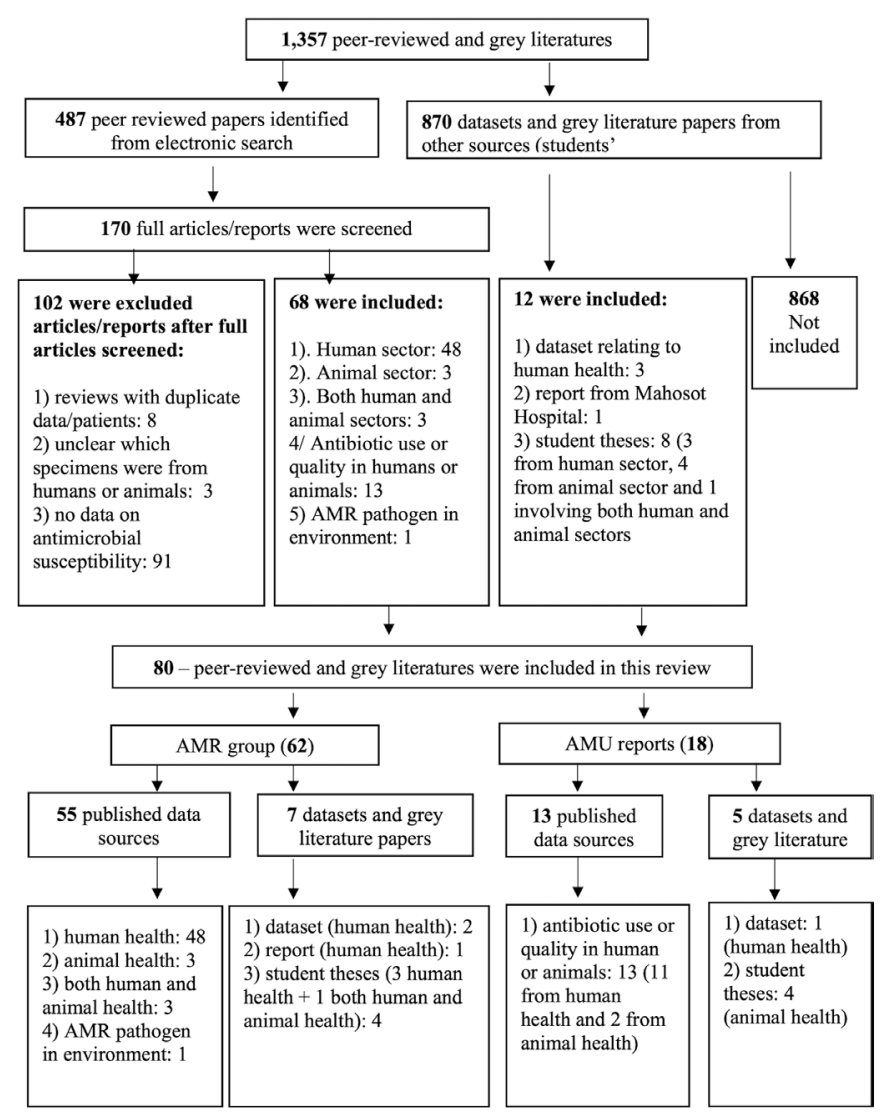

AMR reports: included peer-reviewed and grey literatures with antimicrobial susceptibility data in either human, animal or environment sectors

AMU reports: included peer-reviewed and grey literatures with data of antimicrobial use in either human, animal or environment sectors

Figure 1 Search strategy and selection of studies for the review. AMR, antimicrobial resistance; $A M U$, antimicrobial use.

\section{Evidence for AMR in bacteria infecting humans in Laos}

From 1994 to 2020, S. aureus, E. coli, K. pneumoniae and $S$. Typhi were the most frequently mentioned bacterial pathogens in literature from Laos, mostly from fever aetiology studies with multiple pathogens isolated.

No distinction is made here between communityacquired infections (CAI) and hospital-acquired infections $(\mathrm{HAI})^{7}$ as most available data sources did not include this information. The largest available dataset in this review was from Mahosot Hospital from 2000 to 2016 , which did not include information to be able to identify HAI reliably. However, among 37,443 patients with blood cultures submitted to this laboratory during this period, blood cultures from 32,675 (87\%) were submitted within 48 hours after admission to hospital; which are therefore believed to represent CAI. Blood cultures from 4,396 (12\%) patients were submitted after 48 hours of admission. However, we cannot be sure that these represent HAI because of delays in submitting blood cultures, that only became an accessible service in 2000. In addition, $372(1 \%)$ of patient records lacked dates of admission and/or blood cultures. It will be vital to systematically collect such data to increase our understanding of the comparative antimicrobial susceptibility patterns of both CAI and HAI. ${ }^{8}$

\section{AMR of WHO GLASS target pathogens Escherichia coli}

ESBL-producing $E$. coli were first identified in Laos in 2004 from blood. ${ }^{9}$ Chang et al. reported the trend of ESBL-producing E. coli bacteraemia in Laos from 2010 to 2014, increasing from $7.8 \%(4 / 51)$ to $34.7 \%(17 / 49){ }^{10}$ A larger dataset from Microbiology/LOMWRU laboratory from 2004 to 2016, which included data from Chang et $a l$., showed that the actual proportion increased fivefold from $7 \%(2 / 28)$ in 2004 to $35 \%$ (27/78) in 2016 (figure 3). ${ }^{8}$ Among 280/1,837 inpatients and outpatients at Mahosot Hospital with urinary tract infection (UTI) that yielded significant growth from 2010 to 2011, 194/280 (69\%) grew E. coli. Of these, 28/194 (14\%) were confirmed as ESBL-producing. Only 22/151 (15\%) were resistant to nitrofurantoin, ${ }^{11}$ which is not yet available in Laos. More recent (2017-2018) data on urine cultures from patients with UTI from Mahosot Hospital included 169 E. coli isolates, of which 90 (53\%) were ESBL-producers, and 43 (25\%) were multidrug resistant (MDR-non-susceptible to $\geq 1$ agent in $\geq 3$ antimicrobial categories). ${ }^{12}$ Nitrofurantoin resistance was found in $5 \%$ $(8 / 169) .{ }^{13}$ E. coli carbapenem resistance was first identified in Laos in 2015 from a pus specimen from a Mahosot Hospital inpatient. By December 2020, nine more carbapenem resistant isolates from hospitalised patients had been identified by the Microbiology/LOMWRU laboratory (two from blood). ${ }^{13-15}$ New Delhi Metallobetalactamase (NDM) genes were identified from four. ${ }^{14}$

\section{Klebsiella pneumoniae}

ESBL-producing $K$. pneumoniae were first identified in Laos from blood in 2000. Of 284 K. pneumoniae isolates from blood cultures at Mahosot Hospital from 2000 to 2016, 39 (14\%) were ESBL producers and 20/39 (51\%) were also MDR. ${ }^{12}$ Unlike ESBL-producing E. coli, ESBLproducing $K$. pneumoniae showed no clear time trend over this period and the proportion varied from $3 \%$ to $41 \%$ (online supplemental appendix 5). ${ }^{810}$ Of $32 \mathrm{~K}$. pneumoniae isolates from blood during 2015 and 2016, 18 (56\%) carried the CTX-M-15 gene. ${ }^{16}$ K. pneumoniae carbapenem susceptibility has been tested since 2010. Five resistant isolates were reported from non-blood specimens and one from blood between January 2018 and December 2020. Of these six, only three isolates were tested for carbapenemase and all of them carried NDM genes. ${ }^{13}$

\section{ESBL-producing Enterobacterales carriage/colonisation and colistin resistance studies}

ESBL-producing Enterobacterales stool colonisation has been investigated in Lao communities. ESBL-producing E. coli were found in $25 \%(100 / 397)$ of young children in 12 preschool childcare facilities in the capital city and the wider province of Vientiane. ${ }^{17}$ Of stool specimens from 57 healthy adults in southern Laos, 41 (72\%) contained 


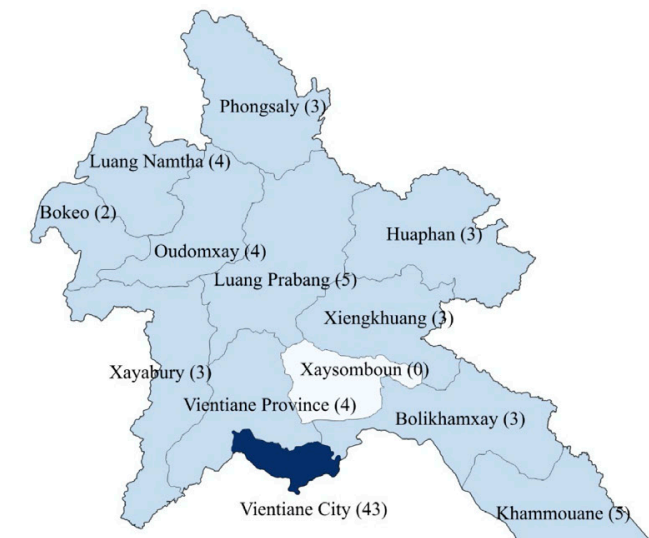

Antimicrobial resistance group

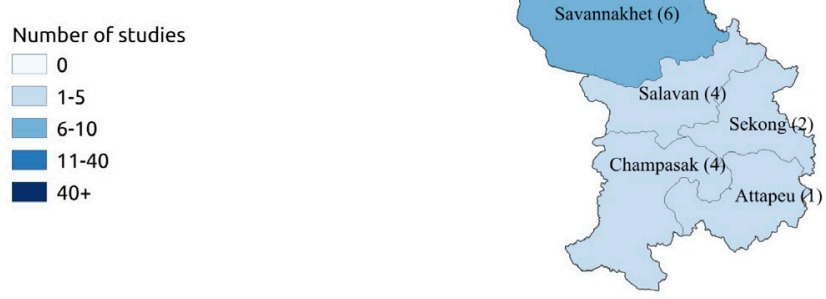

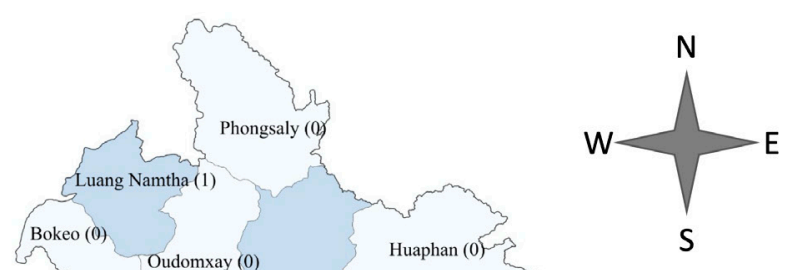

Antimicrobial use group

Number of studies

0

$\square 1-5$

$5+$

Figure 2 Distribution of antimicrobial resistance (AMR), left and antimicrobial use (AMU) data sources, right, in this review. Numbers after each province name (in parentheses) refer to the number of studies relating to either AMR or AMU in Laos. Numbers in parentheses were counted based on number of studies/reports relating to that province. Thirteen and two data sources were not included in the AMR and AMU maps, respectively, because their study sites were not clearly specified.

ESBL-producing E. coli. ${ }^{18}$ In a larger community crosssectional study in a remote village in north-east Laos in 2016, ESBL-producing E. colicolonisation was found in $4 \%$ $(11 / 268)$ of rectal specimens from healthy individuals. ${ }^{19}$ The most recent study published in 2021 demonstrated that 174/236 (74\%) stool specimens collected from 20 healthy European visitors contained ESBL-producing Gram-negative bacteria during their stay in Laos. Of 292

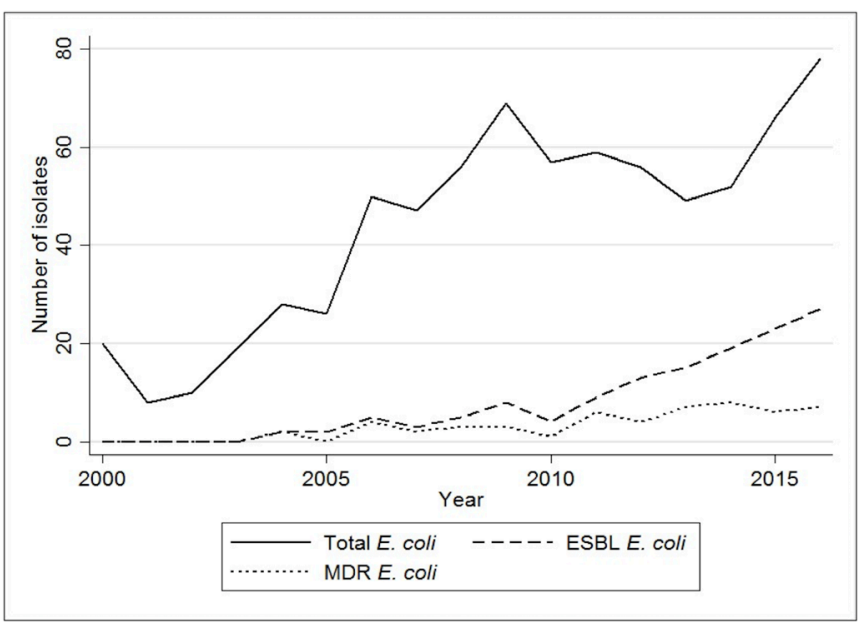

Figure 3 Trends of Escherichia coli, ESBL E. coli and MDR E. coli isolates from blood cultures from 2000 to 2016 at Mahosot Hospital, Vientiane. ESBL, extended spectrum beta lactamase; MDR, multidrug resistant.
ESBL-producing Gram-negative isolates from stool specimens, the CTX-M-15 gene was most frequently identified $(262 / 292(77 \%)) .^{20}$

Although colistin is not available for human use in Laos, it is used for livestock. ${ }^{21}$ Eleven $(6 \%)$ colistin resistant $K$. pneumoniae isolates were reported from 190 stool samples of healthy Lao individuals. Among these 11 isolates, nine different STs were reported and seven $(64 \%)$ had $m g r \mathrm{~B}$ genes without mutations. ${ }^{22}$ Of three isolates from Olaitan et al. ${ }^{22}$ all three carried $m c r-3$ variants, and one carried both $m c r-3$ and $m c r-8 .^{23}$

\section{Staphylococcus aureus}

The first isolation of MRSA ( mесA positive) in Laos was reported in 1997 from the nose of an inpatient, without signs of local infection, in a central Vientiane hospital. ${ }^{24}$ In 2002, the first two MRSA isolates from clinically infected patients were reported. ${ }^{25}$ The first MRSA isolated from blood was identified at Mahosot Hospital in 2017, with 14 subsequent cases by December 2020 for the whole country. S. aureus from non-blood culture specimens during 2012 and 2014 was described in 2017 with Panton-Valentine leukocidin positivity reported in 56/96 (58\%) of isolates. MRSA was found in seven (7\%) isolates including important strains circulating in neighbouring countries, such as ST239-MRSA-III and ST59/952-MRSA-V(T) ${ }^{26}$ More recent data during 2017 and 2018, yielded 95/619 (15\%) MRSA from non-blood culture specimens submitted to 
the Microbiology/LOMWRU laboratory; 7/93 (8\%) were resistant to trimethoprim-sulfamethoxazole, 10/90 (11\%) to ciprofloxacin, 15/91 (16\%) to chloramphenicol, 19/95 $(20 \%)$ to gentamicin and $80 / 94(85 \%)$ to tetracycline. No molecular investigations have been performed. All isolates were fully susceptible to vancomycin but this is not currently available in Laos. ${ }^{13}$

\section{Salmonella Typhi}

A case series of $913 \mathrm{~S}$. Typhibacteraemic patients from Mahosot Hospital (2000-2018) included susceptibility data from diverse fever studies ${ }^{27-32}$; only $12 / 854$ isolates $(1.4 \%)$ were resistant to quinolones (either ciprofloxacin or nalidixic acid or both), 71/893 (8\%) to ampicillin, 70/865 $(8 \%)$ to chloramphenicol, $67 / 885(7.6 \%)$ to trimethoprimsulfamethoxazole and 59/848 (7\%) were MDR. Ceftriaxone is the first choice of complicated typhoid treatment in Laos, and $91 \%(739 / 815) S$. Typhi were highly susceptible to ceftriaxone. There has been no report of ceftriaxone resistant $S$. Typhi since 2009..$^{33}$ Fluoroquinolone resistant $S$. Typhi in Laos are rare and treatment with fluoroquinolones are still likely to remain efficacious, as demonstrated in a clinical trial from 2002. ${ }^{32}$ Azithromycin is not routinely tested against $S$. Typhi in Laos. Azithromycin minimum inhibitory concentration (MIC) of $\leq 16 \mu \mathrm{g} / \mathrm{mL}$ was widely described as statistically clinical response for uncomplicated $S$. Typhi infection, 232/1,460 S. Typhi isolates from Laos were described in Parry et al. Of 1,460 isolates, $99.5 \%$ showed MIC of $\leq 16 \mu \mathrm{g}$ / $\mathrm{mL} .{ }^{34}$

\section{Non-typhoidal Salmonella}

Antimicrobial susceptibility of non-typhoidal Salmonella spp. (NTS) has not been documented in detail in Laos. Between 2000 and 2012, 168 NTS (63 from blood and 105 from faecal specimens) were isolated at Mahosot Hospital. NTS isolated from faecal samples were more frequently resistant to ampicillin and trimethoprim-sulfamethoxazole than those isolated from blood $(\mathrm{p}<0.05)$. In contrast, NTS resistant to ciprofloxacin were found more frequently in blood than in stool $(\mathrm{p}<0.001) .{ }^{35}$ In 2017, of 519 stool cultures reported from Mahosot Hospital, 35 grew NTS. Of these, $29 \%$ were resistant to azithromycin, $26 \%$ to trimethoprim-sulfamethoxazole, $23 \%$ to ciprofloxacin, $23 \%$ to ceftriaxone, $20 \%$ to chloramphenicol, $9 \%$ to ampicillin and $6 \%$ to nalidixic acid. ${ }^{13}$

\section{Shigella spp.}

Shigella spp. infection is also not well documented in Laos, with only two published reports. ${ }^{36}{ }^{37}$ Forty-five Shigella isolates from Lao patient stools during 2006 and 2012, comprised 35 S. flexneri, nine $S$. sonnei and one $S$. boydii; and 14/45 (31\%) were resistant to nalidixic acid and $34 / 45(76 \%)$ were MDR. All isolates were susceptible to ceftriaxone and azithromycin. ${ }^{37}$

\section{Neisseria gonorrhoeae}

Four studies of Neisseria gonorrhoeae susceptibility in Laos have been published ${ }^{38-41}$ during this review period. In 2003, 1/131 (0.8\%) N. gonorrhoeae isolates from 1937 genital clinical specimens was reported to be resistant to ceftriaxone, along with one further $(0.8 \%)$ isolate with decreased spectinomycin susceptibility. ${ }^{38}$ Although no such strains have been described in subsequent studies, high frequencies of resistance to tetracycline, penicillin and ciprofloxacin were reported. ${ }^{39-41}$ More recent data from 2011 to 2015 included $158 \mathrm{~N}$. gonorrhoeae isolated from 12281 genital samples from patients at Mahosot Hospital; all were susceptible to ceftriaxone and spectinomycin. However, $99 \%, 90 \%$ and $85 \%$ were resistant to tetracycline, penicillin and ciprofloxacin, respectively. ${ }^{41}$ $N$. gonorrhoeae azithromycin susceptibility has not been tested for in Laos.

\section{Streptococcus pneumoniae}

Two publications have described Streptococcus pneumoniae infections in Laos, ${ }^{42} 43$ mainly focused on central nervous system infections, using overlapping datasets from Mahosot Hospital. Moore et al. described 2/23 isolates of S. pneumoniae from 2003 to 2009 from blood and cerebrospinal fluid (CSF), with reduced susceptibility to penicillin (both had minimum inhibitory concentrations $\geq 0.12 \mu \mathrm{g} / \mathrm{mL}(0.39$ and $0.125 \mu \mathrm{g} / \mathrm{mL}$, respectively) ). ${ }^{42}$ A larger dataset from 2003 to 2011 from the same hospital revealed that $3 / 11$ isolates of $S$. pneumoniae from CSF had reduced susceptibility to penicillin. ${ }^{43}$

\section{Acinetobacter baumannii}

The first report on the susceptibility of A. baumannii in Laos was in 2019, describing 22 carbapenem-resistant A. baumannii isolates from 2017 (19 from endotracheal aspirates, two wound swabs and one blood culture). Of these, 18 (82\%) were susceptible to amikacin but all were resistant to imipenem, ceftazidime, ciprofloxacin and tetracycline. ${ }^{1415}$

\section{Mycobacterium tuberculosis}

The first multi-centre study of Mycobacterium tuberculosis susceptibility was conducted in 2010 in three Lao provinces. Of 87 (84\%) M. tuberculosis cultured from 104 sputum samples, $8(9.2 \%)$ were resistant to one or more antituberculous agents, of which seven were monoresistant to isoniazid (INH) and one was MDR that was subsequently found to be extensively drug resistant $\mathrm{TB}^{4}{ }^{44}$ Somphavong et al. characterised the genetics of these $M$. tuberculosis isolates from the national survey conducted from July 2010 to December 2011. Of 202 isolates, the East African-Indian lineage was the most common identified in all provinces, except Xiengkhuang. The Beijing lineage was found mostly in the northern and central Laos. ${ }^{45}$

From 2016 to 2017, of 1,006 sputum samples submitted to 42 TB laboratories throughout the country, 946 (94\%) were GeneXpert MTB/RIF positive (897 (95\%) new cases and $49(5 \%)$ previously treated cases). Of these, $820(87 \%)$ samples were available for AST. Seventy $(8.5 \%)$ were resistant to antituberculous agents used for first-line treatment (either INH, rifampicin, ethambutol 
or streptomycin). Rifampicin resistance was found in $11 / 897(1.2 \%)$ new cases and 2/49 (4\%) previously treated cases. Five $(0.6 \%$ of 820 isolates $)$ were MDR $(4 / 776$ in new cases and $1 / 44$ previously treated cases). No second-line TB drug (kanamycin, capreomycin and ofloxacin) resistance was reported. ${ }^{46}$

\section{AMR of other bacteria of local and regional importance}

Diverse other infectious diseases are common in Laos, ${ }^{47}$ but there are very limited AMR data available. Intrinsic AMR is important for melioidosis and rickettsial diseases, reducing the spectrum of efficacious antibiotic therapy. Burkholderia pseudomallei is a common cause of bacteraemia in Laos and necessitates a prolonged treatment course of ceftazidime followed by co-trimoxazole, but acquired AMR has not become a clinical problem. ${ }^{48}$ Zoonoses such as rickettsial pathogens and leptospirosis are common causes of febrile diseases in Laos; the limited evidence also suggests that acquired AMR in these infections is not yet a major issue ${ }^{48} 49$ (online supplemental appendix 2).

\section{Evidence of AMR in bacteria in animals}

Data on food production animal AMR in Laos are very limited, with the majority on Salmonella spp. ${ }^{50-54}$ followed by E. coli, ${ }^{195355}$ with sparser data for Enterococcus faecalis and Enterococcus faecium ${ }^{56}$ (online supplemental appendix 3). The proportion of Salmonella spp. contaminating pork/pig carcasses, beef and buffalos at slaughterhouses and retail markets in central and southern Laos ranged from $39 \%$ to $93 \%$ from 2007 to 2017 , with the proportion of quinolone resistance at $2 \%-25 \% .^{50-53}$ Food production animals carrying ESBL-producing E. coli were also described in a remote rural village in Xiengkhuang Province in northern Laos. Of 252 food production animal rectal swabs, $21(8 \%)$ carried ESBL-producing E. coli, even though these food production animals were mostly born in the village and fed with local products. ${ }^{19}$ Four colistin-resistant E. coli isolates from pigs have been described and also identified in a Lao boy with the same novel ST as his family's pigs. ${ }^{55}$

\section{Evidence of AMU in Laos}

AMU in Laos has also not been widely studied. Here we summarise ten AMU data sources for humans; one dataset and nine publications. Information on animal use is very limited, with only four veterinary student theses, one published report and one publication (online supplemental appendix 4).

\section{AMU in humans}

Antimicrobials were prescribed for between $45 \%$ and $70 \%$ of inpatients in Lao hospitals from 2004 to $2018 . .^{57-60}$ Doctors stated that they mainly prescribed antibiotics according to the National Standard Treatment Guidelines (STG), advice from their peers or from more experienced colleagues. ${ }^{57}$ Until recently these guidelines have not included detailed recommendations for antimicrobial prescribing for the diversity of Lao infectious diseases, but detailed $\mathrm{MoH}$ antimicrobial guidelines have now been released. ${ }^{61} 62$

From 2017 to 2018, five hospitals (Mahosot Hospital, Xiengkhuang, Luang Namtha, Vientiane and Salavan Provincial Hospitals) participated in the Global Point Prevalence Survey (http://www.global-pps.com) (Chansamouth $e t$ al. in prep). Of 1,981 hospitals charts screened, $1,386(70 \%)$ patients received antimicrobial(s); the proportion of antimicrobial prescriptions was higher in provincial hospitals than in central hospitals at $72 \%$ vs $66 \%(\mathrm{p}=0.003)$. Of 1,386 patients prescribed antimicrobial(s), 596 (43\%) were prescribed two or more. Of all prescriptions, beta-lactams accounted for 1,272/2,052 (62\%) $(67 \%$ cephalosporins, $30 \%$ penicillins, $2 \%$ betalactam/beta-lactamase inhibitors and $1 \%$ carbapenems), metronidazole 287/2,052 (14\%) and aminoglycosides 226/2,052 (11\%) (online supplemental appendix 6 and 7) ${ }^{59}$ The appropriateness of the antimicrobials was not assessed. However, in Luang Namtha Provincial Hospital from 2008 to $2010,560 / 1,095(51 \%)$ patients received antibiotics but only 39/560 (7\%) of these were considered appropriate. ${ }^{27}$ In an analysis of antibiotic use in $2019,397 / 413(94 \%)$ of patients were prescribed antibiotics for upper respiratory infection and 8/164 (4.9\%) for common colds. First-line antibiotics based on Lao National STG (amoxicillin, ampicillin, erythromycin and penicillin $\mathrm{V}$ ) were the most commonly prescribed. ${ }^{60}$

A large survey in Salavan Province between 2017 and 2018 revealed that among 796 participants from communities, 39\% claimed that they used antibiotics during their previous illness and $22 \%$ admitted that they used antibiotics without indications or from informal sources. ${ }^{63}$ 'Ampi' or ampicillin was the most commonly mentioned antibiotic, by $76 \%$ of 775 survey participants, and wound treatment $(44 \%)$ was the main reason for antibiotic use in Salavan in the same period. ${ }^{64}$ In Vientiane and Champasak Provinces, among 500 adults who self-medicated with antimicrobials for reproductive tract infections during the previous year, ampicillin (165 (33\%)) was the most frequently used, and more than $50 \%$ used unrecommended combined medications. ${ }^{65}$ Participants claimed that seeing a doctor might not be necessary because they could easily access antibiotics without prescriptions and they obtain the same antibiotic that they had used previously. ${ }^{66}$ Pharmacies were common sources of antibiotics for self-medication $(85 \%(326 / 384))$ in three districts of Vientiane City in 2017. ${ }^{67}$ Moreover, some participants could access antibiotics from local grocery shops $(69 / 384(18 \%)) .{ }^{67}$ The use of antibiotics prior to hospital consultation, as indicated by antibiotic activity in urine, was significantly higher in children $(60 \%)$ than in adults $(47 \%)(\mathrm{p}<0.0001) .{ }^{68}$ Ampicillin, amoxicillin, penicillin and tetracycline were frequently chosen for selfmedication before deciding to go to hospital. ${ }^{316568}$

More than one-third of Lao doctors from 25 public hospitals in four provinces thought that antibiotics in their hospitals were of poor quality. ${ }^{57}$ However, for consumers the cost of medications was of greater concern 
than their quality. ${ }^{57} 69$ Indeed, issues with substandard and falsified antibiotics have been described in Laos and these are likely to be globally neglected drivers of AMR and impaired patient outcome..$^{70}$

\section{AMU in animals}

The use of antibiotics in Lao livestock is thought to be common but is not adequately supervised and there are few available data. ${ }^{72}$ Although no antimicrobial agents for animals have been registered with the Lao Department of Livestock and Fisheries (DLF), some antibiotics and vaccines are available at the DLF pharmacy and at private animal clinics. Most available publications/ reports on AMU in animals focused on targeted treatment rather than growth promotion. Between 2002 and 2017, four students (out of 872) at the Department of Veterinary Medicine and DLF, National University of Laos studied the use of antibiotics in animals. These four theses focused on the effects of the use of cephalexin, amoxicillin, clavulanate, oxytetracycline, sulphonamides, enrofloxacin and gentamicin for treating infections in domestic dogs, with no studies on livestock. ${ }^{74-77}$ Among domestic elephants in Xayabury Province, antibiotics were used for treatment of abscesses, superficial wounds and eye problems. Oxyblue spray or penicillin-streptomycin were commonly used for abscesses and enrofloxacin for UTIs. As the country lacks provincial veterinary diagnostic laboratories, most infectious disease treatments in animals are empirical. ${ }^{78} \mathrm{~A}$ recent article on the antibiotic supply chain in the animal sector in Laos, but outside this review period, ${ }^{21}$ found that of 96 chicken farms and 96 pig farms, $49 \%$ and $60 \%$, respectively, claimed that they gave antibiotics to their animals. Of 29 chicken farms with antibiotics found during the survey, tetracycline was the most commonly found, in 10/29 (34\%) of chicken farms. Amoxicillin was the most common in pig farms at $17 / 73(23 \%) .{ }^{21}$ There have not been reports or data on AMU in aquaculture, nor on AMR among fish bacterial pathogens in Laos.

\section{AMR pathogens in the environment}

There is almost no information on contamination of the Lao environment with AMR pathogens. In 2003, drugresistant Vibrio cholerae from surface water samples was sought in thirteen tributaries of the Mekong River in cholera-epidemic areas in Laos after an outbreak in 2000. Twenty-two non-O1 and non-O139 $V$. cholerae isolates were tested against polymyxin $\mathrm{B}$, tetracycline, ampicillin, ampicillin-clavulanic acid, erythromycin, nalidixic acid, chloramphenicol, trimethoprim-sulfamethoxazole and streptomycin. Polymyxin B resistance was found in 2/22 (9\%) and ampicillin-resistance in 15/22 (68\%), although isolates were susceptible to other tested antibiotics. ${ }^{79}$

\section{DISCUSSION}

There is little evidence on AMR awareness and how to engage with the public, health workers and policy makers in Laos. ${ }^{5758} 80$ A large multicentre study of AMR awareness across 12 WHO member states (two from each WHO region) showed that the level of knowledge and awareness around appropriate antibiotic use varies from country to country. ${ }^{81}$ Many participants $(32 \%-62 \%)$ thought that they should stop taking antibiotics when they felt better. Nearly half (43\%) thought that they could buy the same antibiotics again if they had made them feel better during a previous illness and $44 \%$ believed that AMR is only a problem in people who take antibiotics regularly. ${ }^{81}$

Antimicrobial misuse in animal husbandry is thought to be one of the most important AMR drivers. However, we could not find any data on antibiotic use in livestock and aquaculture in Laos during this review period. There is an urgent need in Laos to systematically collect and analyse data on antibiotic use, consumption and resistance in humans, animals and the environment. Innovative public engagement strategies will be needed to raise awareness of optimal AMU and the risks of AMR. ${ }^{82}$

\section{AMR data compared with adjacent countries}

Data on AMR among GLASS target pathogens are not well documented in Laos compared with neighbouring countries (tables 1 and 2). Most publications investigated causes of fever rather than specific pathogens and their susceptibility patterns. In Laos, current evidence suggests that the main concern is an increase in ESBL-producing E. coli. Community-acquired and hospital-acquired bacteraemia have not been well distinguished and further work to focus on this will be important. There is evidence for the emergence of carbapenem-resistant $E$. coli and $K$. pneumoniae, and colistin-resistant $K$. pneumoniae in Laos. Carbapenems and amikacin are the only antibiotic of choice for ESBL treatment available in Laos. It would be of great concern if combined carbapenem and colistin resistance were to emerge (colistin is not currently available in Laos for human use) as this would lead to infections that are essentially untreatable in Laos.

MRSA is a universal public health concern, and well documented in neighbouring countries. In 2021, Vu et al reported that 3,302/4,515 (73\%) of $S$. aureus in all specimen types from 13 hospitals in Vietnam during 2016 and 2017 were MRSA. In addition, of 674 S. aureus isolates from blood or CSF, $71 \%$ (476) were MRSA. ${ }^{83}$ In Thailand, the proportion of MRSA was high among patients with hospital-acquired $S$. aureus bacteraemia $(48 \%, \mathrm{n}=441)$, and $7 \% \quad(\mathrm{n}=1145)$ among community-acquired bacteraemia patients. The proportion of MRSA not susceptible to vancomycin (Vancomycin-resistant Staphylococcus aureus (VRSA) ) was $0.4 \%(6 / 1,380) .{ }^{84}$ MRSA bacteraemia was also common in a large bacteraemia study $(\mathrm{n}=4,833$ adult patients) in Phnom Penh, Cambodia; 22\% (10/46) of $S$. aureus were MRSA but all were susceptible to vancomycin. ${ }^{85}$ In contrast, in Laos only six MRSA were isolated from blood during this review period; the number of MRSA in non-blood specimens was higher (15\%). VRSA has not yet been reported in Laos. 
Table 1 Lao antimicrobial resistance frequency for Escherichia coli, isolated from blood and cerebrospinal fluid, compared with equivalent data from adjacent countries

\begin{tabular}{|c|c|c|c|c|c|}
\hline \multirow[b]{2}{*}{ E. coli } & \multirow{2}{*}{$\begin{array}{l}\text { Laos }^{*} \\
\text { Blood; n=750 } \\
(2000-2016)\end{array}$} & \multicolumn{2}{|l|}{${\text { Vietnam } t^{83}}^{8}$} & \multirow{2}{*}{$\begin{array}{l}\text { Cambodia }^{85} \\
\text { Blood; } n=130 \\
(2007-2010)\end{array}$} & \multirow{2}{*}{$\begin{array}{l}\text { Thailand }^{84} \\
\text { Blood; } n=4,278 \\
(2004-2010)\end{array}$} \\
\hline & & $\begin{array}{l}\text { All specimens; } \\
\mathrm{n}=9,092 \\
(2016-2017)\end{array}$ & $\begin{array}{l}\text { Blood and CSF; } \\
n=1,535 \\
(2016-2017)\end{array}$ & & \\
\hline ESBL & 135/693 (19\%)‡ & $4,085 / 6,953$ (59\%) & $655 / 1,107(59 \%)$ & $62(48 \%)$ & \\
\hline Amikacin & $2 / 135(1 \%)$ & $4,188 / 8,785$ (48\%) & $637 / 1,471(43 \%)$ & $5(4 \%)$ & $130 / 3,408(4 \%)$ \\
\hline Gentamicin & 120/675 (18\%) & & & 73 (56\%) & 903/4228 (21\%) \\
\hline Cefotaxime & - & $5,441 / 8,195$ (66\%) & $931 / 1,402(66 \%)$ & $67(52 \%)$ & $885 / 3,892(23 \%)$ \\
\hline Ceftazidime & $69 / 180(38 \%)$ & & & $47(36 \%)$ & $721 / 3,817$ (19\%) \\
\hline Chloramphenicol & $148 / 579(26 \%)$ & - & - & - & 18/72 (25\%) \\
\hline Ciprofloxacin & $74 / 231(32 \%)$ & $5,813 / 8,682(67 \%)$ & $953 / 1,475(65 \%)$ & $85(65 \%)$ & $1,120 / 3,836(29 \%)$ \\
\hline Co-amoxiclav & $94 / 673(14 \%)$ & $1,476 / 3,251(45 \%)$ & 180/577 (31\%) & $64(49 \%)$ & 1,139/3,910 (29\%) \\
\hline Imipenem & - & $961 / 8,830(11 \%)$ & $116 / 1,483(8 \%)$ & - & $6 / 3,179(<1 \%)$ \\
\hline Meropenem & $0 / 144(0)$ & & & 0 & $2 / 2,546(<1 \%)$ \\
\hline $\begin{array}{l}\text { Trimethoprim- } \\
\text { sulfamethoxazole }\end{array}$ & 429/704 (61\%) & $5,704 / 7,843$ (73\%) & $935 / 1,377(68 \%)$ & $214(95 \%)$ & $2,257 / 3,799(59 \%)$ \\
\hline
\end{tabular}

Note that there are a paucity of published data and the year ranges differ between countries.

*LOMWRU data held by Microbiology/LOMWRU, Mahosot Hospital.

†Antimicrobial testing data from Vu et al. ${ }^{83}$ showed as a class of antibiotic rather than antibiotic agent.

łDuration: 2004-2016; ESBL-producing E. coli in 2016 only was 27/78 (35\%) in blood. However, ESBL-producing E. coli in urine (2017-2018) was $90 / 169(53 \%)$.

CSF, cerebrospinal fluid; ESBL, extended spectrum beta lactamas; LOMWRU, Lao-Oxford-Mahosot Hospital-Wellcome Trust Research Unit.

These data suggest that Laos is thus surrounded by countries with an apparently higher frequency of antibiotic resistant pathogens. Paucity of AMR and AMU data puts Laos at a disadvantage in terms of understanding and controlling antibiotic resistance. However, the Lao data suggest that key bacterial pathogens within the country generally have a lower frequency of AMR than adjoining countries, especially $S$. aureus and $S$. Typhi, but that Laos has a severe and growing problem with ESBL production in E. coli, not only in patients but also in carriage by healthy individuals. ${ }^{17-19}$

Although there are no data to inform this objectively, Laos' apparently lower burden of AMR may have resulted from historically lower antibiotic access and use due to

Table 2 Lao antimicrobial resistance frequency for Staphylococcus aureus, isolated from blood and cerebrospinal fluid, compared with equivalent data from adjacent countries

\begin{tabular}{|c|c|c|c|c|c|}
\hline \multirow[b]{2}{*}{ S. aureus } & \multirow{2}{*}{$\begin{array}{l}\text { Laos }^{89} \\
\text { Blood; } n=200 \\
(2000-2011)\end{array}$} & \multicolumn{2}{|l|}{ Vietnam $^{\star 83}$} & \multirow{2}{*}{$\begin{array}{l}\text { Cambodia }^{85} \\
\text { Blood; } n=46 \\
(2007-2010)\end{array}$} & \multirow{2}{*}{$\begin{array}{l}\text { Thailand }^{84} \\
\text { Blood; } n=1,881 \\
(2004-2010)\end{array}$} \\
\hline & & $\begin{array}{l}\text { All specimens; } \\
\mathrm{n}=4,833 \\
(2016-2017)\end{array}$ & $\begin{array}{l}\text { Blood and CSF; } \\
n=715 \\
(2016-2017)\end{array}$ & & \\
\hline MRSA & $\dagger$ & $3,302 / 4,515(73 \%)$ & 476/674 (71\%) & $10 / 46(22 \%)$ & $357 / 389(92 \%)$ \\
\hline Ciprofloxacin & - & 1,720/4,619 (37\%) & 297/689 (43\%) & - & $7 / 63(11 \%)$ \\
\hline Erythromycin & $70 / 180$ (39\%) & $3,861 / 4,661(83 \%)$ & $545 / 639(79 \%)$ & $24 / 46$ (52\%) & $436 / 1,785(24 \%)$ \\
\hline Gentamicin & $2 / 166(1 \%)$ & 1,674/4,090 (41\%) & 294/637 (46\%) & - & 106/719 (15\%) \\
\hline Penicillin & 158/170 (93\%) & $2,347 / 2,400(98 \%)$ & 490/504 (97\%) & 45/46 (98\%) & - \\
\hline Tetracycline & $39 / 81(48 \%)$ & - & & 24/46 (52\%) & - \\
\hline $\begin{array}{l}\text { Trimethoprim- } \\
\text { sulfamethoxazole }\end{array}$ & $16 / 151(11 \%)$ & $1021 / 4158(25 \%)$ & $233 / 661$ (35\%) & $11 / 46$ (24\%) & $341 / 1,828(19 \%)$ \\
\hline Vancomycin & 0/52 (0) & 45/2,680 (2\%)‡ & 7/565 (1\%) & - & $6 / 1,380(<1 \%)$ \\
\hline
\end{tabular}

Note that there are a paucity of published data and the year ranges differ between countries.

${ }^{*}$ Antimicrobial testing data from Vu et $a{ }^{83}$ showed as a class of antibiotic rather than antibiotic agent.

†The first MRSA bacteraemia in Laos was identified in 2017.

$\ddagger$ Resistant and intermediate.

CSF, cerebrospinal fluid; MRSA, methicillin-resistant Staphylococcus aureus. 
being relatively isolated until $\sim 20$ years ago and financially impoverished, and there being only a small accessible commercial antibiotic market in the past. However, the apparent lower AMR burden may also partly reflect the paucity of systematic surveillance and research. Recent initiatives such as support for AMR and AMU surveillance by $\mathrm{MoH}$ through the Fleming Fund ${ }^{86}$ and $\mathrm{KOICA}^{87}$ aim to increase the availability of actionable data from across the country. Related initiatives such as the development of updated MoH infectious diseases treatment guidelines, AMR and AMU dashboards (https://www.youtube.com/ watch? $v=\mathrm{QELwHIPsKw} 4$ ) will facilitate policy engagement and enhance antibiotic stewardship and AMR prevention, detection and pharmacist-led antimicrobial stewardship programmes and empowering hospital drugs and therapeutics committees could be explored as interventions to enhance AMU within healthcare facilities. Although it is illegal to sell antibiotics without a prescription in Laos, buying antibiotics without a prescription is anecdotally common and interventions to improve this situation will be vital. Recent interventions such as detailed antimicrobial treatment guidelines and inpatient and outpatient patient AMU data collection will provide an infrastructure for improving and monitoring AMU.

\section{Limitations}

Data on AMU and AMR in Laos are sparse and scattered and are mostly from Vientiane City. The majority (49\%) of data sources on AMR and AMU were from one site, at LOMWRU/ Mahosot Hospital. Though the number of publications on infectious diseases in Laos has greatly increased since $2000,{ }^{4788}$ there are few articles that specifically describe the AMU and AMR situations in Laos. Fifteen percent of the data sources on AMR and AMU in this review were not peer-reviewed. The majority of data were from epidemiological studies, cross-sectional studies and three large microbiology datasets from LOMWRU/ Mahosot Hospital from 2000 to 2020.

\section{CONCLUSION}

If AMR is, as these data suggest, of relatively low frequency in Laos for some GLASS target pathogens, it gives the country a key window of opportunity to act quickly to implement strategies to protect the population and health system from a worsening situation, through preventing, detecting and responding to AMR threats. However, the paucity of systematic surveillance of AMR and AMU in Laos must be taken into account when interpreting the low frequency of AMR in this setting. Enhanced coordinated and systematic AMU and AMR surveillance across the country is needed. Laos needs enhanced regulation of antibiotic availability, without compromising appropriate access, and strengthened antibiotic stewardship in health facilities and the community for systematic control and rationalisation of AMU, in order to prevent resistance before it spreads and becomes harder to manage. Strategies should also be put in place to control the use of colistin in livestock, as colistin is currently a last resort for treating key drug resistant pathogens. Specific strategies should also be developed to prevent a rise in $S$. aureus and $S$. Typhi resistance to key antibiotics and to reduce the high prevalence of ESBL-producing Enterobacterales.

\section{Author affiliations}

${ }^{1}$ Microbiology Laboratory, Mahosot Hospital, Vientiane, Lao People's Democratic Republic

${ }^{2}$ Lao-Oxford-Mahosot Hospital-Wellcome Trust Research Unit (LOMWRU), Mahosot Hospital, Vientiane, Lao People's Democratic Republic

${ }^{3}$ Centre for Tropical Medicine and Global Health, Nuffield Department of Medicine, University of Oxford, Oxford, UK

${ }^{4}$ Faculty of Postgraduate Studies, University of Health Sciences, Vientiane, Lao People's Democratic Republic

${ }^{5}$ Faculty of Infectious and Tropical Diseases, London School of Hygiene and Tropical Medicine, London, UK

${ }^{6}$ Department of Communicable Disease Control, Ministry of Health, Vientiane, Lao People's Democratic Republic

${ }^{7}$ Department of Livestock and Fisheries, Ministry of Agriculture, Vientiane, Lao People's Democratic Republic

${ }^{8}$ Department of Food and Drug, Ministry of Health, Vientiane, Lao People's Democratic Republic

${ }^{9}$ Infectious Disease Center, Mahosot Hospital, Vientiane, Lao People's Democratic Republic

${ }^{10}$ Setthathirath Hospital, Vientiane, Lao People's Democratic Republic

${ }^{11}$ Mahidol Oxford Tropical Medicine Research Unit (MORU), Faculty of Tropical

Medicine, Mahidol University, Bangkok, Thailand

${ }^{12}$ Cambodia Oxford Medical Research Unit, Siem Reap, Cambodia

${ }^{13}$ Oxford University Clinical Research Unit, Hanoi, Viet Nam

\section{Twitter Paul Turner @PaulTurnerMicro}

Acknowledgements We would like to thank the late Dr Rattanaphone Phetsouvanh, Dr Phouthavanh Inlorkham and Dr Kongmany Nammavongmixay from the Food and Drug Department, Ministry of Health, Mr Sithixay Kaylath from the Faculty of Agriculture, National University of Laos, Dr Khamsay Dethleuxay and Ms Aphone Visathep from Mahosot Hospital, Dr Manichanh Thongsana from Mittaphab Hospital to support in finding non-peer-reviewed data, Dr Risara Jaksuwan for assisting with obtaining antimicrobial susceptibility data from the Mahosot Microbiology/LOMWRU and Hellen Gelband and Jyoti Joshi from GARP for their support of this review.

Contributors VC, MM, RP, HRvD and PN conceptualised and designed the study. VC, DD, TR, VD, PI, SK, BK, EAA and PN conducted the search and data extraction. VC wrote the first draft. All authors reviewed and provided substantial inputs and feedbacks for subsequent draft. All authors have read and approved the final version of the manuscript. VC and PN critically reviewed the overall content. VC was a guarantor.

Funding This review was funded by GARP, The Center for Disease Dynamics, Economics and Policy (CDDEP) and partly by the Wellcome Trust (Grant number $214207 / Z / 18 / Z$ ). For the purpose of open access, the author has applied a CC BY public copyright licence to any Author Accepted Manuscript version arising from this submission.

Map disclaimer The inclusion of any map (including the depiction of any boundaries therein), or of any geographic or locational reference, does not imply the expression of any opinion whatsoever on the part of BMJ concerning the legal status of any country, territory, jurisdiction or area or of its authorities. Any such expression remains solely that of the relevant source and is not endorsed by BMJ. Maps are provided without any warranty of any kind, either express or implied.

Competing interests None declared.

Patient consent for publication Not applicable.

Provenance and peer review Not commissioned; externally peer reviewed.

Data availability statement Data may be obtained from a third party and are not publicly available. All data relevant to the study are included in the article or uploaded asonline supplemental information.

Supplemental material This content has been supplied by the author(s). It has not been vetted by BMJ Publishing Group Limited (BMJ) and may not have been 
peer-reviewed. Any opinions or recommendations discussed are solely those of the author(s) and are not endorsed by BMJ. BMJ disclaims all liability and responsibility arising from any reliance placed on the content. Where the content includes any translated material, BMJ does not warrant the accuracy and reliability of the translations (including but not limited to local regulations, clinical guidelines, terminology, drug names and drug dosages), and is not responsible for any error and/or omissions arising from translation and adaptation or otherwise.

Open access This is an open access article distributed in accordance with the Creative Commons Attribution 4.0 Unported (CC BY 4.0) license, which permits others to copy, redistribute, remix, transform and build upon this work for any purpose, provided the original work is properly cited, a link to the licence is given, and indication of whether changes were made. See: https://creativecommons.org/ licenses/by/4.0/.

\section{ORCID iDs}

Vilada Chansamouth https://orcid.org/0000-0003-3049-784X

Mayfong Mayxay https://orcid.org/0000-0002-6056-4516

David AB Dance https://orcid.org/0000-0001-9189-7244

Tamalee Roberts http://orcid.org/0000-0001-8599-737X

Nicholas PJ Day https://orcid.org/0000-0003-2309-1171

Paul Turner http://orcid.org/0000-0002-1013-7815

Elizabeth A Ashley http://orcid.org/0000-0002-7620-4822

H. Rogier van Doorn https://orcid.org/0000-0002-9807-1821

Paul N Newton http://orcid.org/0000-0002-4608-6431

\section{REFERENCES}

1 WHO. World Health Organization. Global action plan on antimicrobial resistance. World Health Organization, 2015. https://www.who.int/ antimicrobial-resistance/publications/global-action-plan/en/

2 Holmes AH, Moore LSP, Sundsfjord A, et al. Understanding the mechanisms and drivers of antimicrobial resistance. Lancet 2016;387:176-87.

3 McKenna M. We need antibiotics. They're not profitable to make. Who pays? 2015. Available: https://www.nationalgeographic.com/ science/article/oneill-amr-3 [Accessed 09 Apr 2021].

4 O'Neill J. Tackling drug-resistant infections globally: final report and recommendations. The review on antimicrobial resistance 2016. Available: https://amr-review.org/sites/default/files/160525_Final\% 20paper_with\%20cover.pdf [Accessed 09 Apr 2021].

5 WHO. Global antimicrobial resistance surveillance system (glass) report: early implementation 2020. Geneva: World Health Organization, 2020. https://apps.who.int/iris/bitstream/handle/ 10665/332081/9789240005587-eng.pdf?ua $=1$

6 Cusack TP, Ashley EA, Ling CL, et al. Impact of CLSI and EUCAST breakpoint discrepancies on reporting of antimicrobial susceptibility and AMR surveillance. Clin Microbiol Infect 2019;25:910-1.

7 Hongsuwan M, Srisamang P, Kanoksil M, et al. Increasing incidence of hospital-acquired and healthcare-associated bacteremia in northeast Thailand: a multicenter surveillance study. PLoS One 2014:9:e109324.

8 Microbiology/LOMWRU. Data from: undetermined infections (UI) 2000-2016. Vientiane, Laos: Microbiology Laboratory/Lao-Oxford Mahosot Hospital Wellcome Trust Research Unit (LOMWRU), Mahosot Hospital, 2016.

9 Stoesser N, Crook DW, Moore CE, et al. Characteristics of CTX-M ESBL-producing Escherichia coli isolates from the Lao People's Democratic Republic, 2004-09. J Antimicrob Chemother 2012;67:240-2.

10 Chang K, Rattanavong S, Mayxay M, et al. Bacteremia caused by extended-spectrum beta-lactamase-producing Enterobacteriaceae in Vientiane, Lao PDR: a 5-year study. Am J Trop Med Hyg 2020;102:1137-43.

11 Phakhounthong K, Sivanthong P, Sisomboun P. Etiological pathogens and their antibiotic susceptibility pattern among adult patients with urinary tract infections at Mahosot Hospital. University Library: University of Health Sciences, 2012.

12 Magiorakos A-P, Srinivasan A, Carey RB, et al. Multidrug-resistant, extensively drug-resistant and pandrug-resistant bacteria: an international expert proposal for interim standard definitions for acquired resistance. Clin Microbiol Infect 2012;18:268-81.

13 Microbiology/LOMWRU. Data from: laboratory information management system (LIMS) 2017-2020. Vientiane, Laos: Microbiology Laboratory/Lao-Oxford Mahosot Hospital Wellcome Trust Research Unit (LOMWRU), Mahosot Hospital, 2020.

14 Cusack T-P, Phimolsarnnousith V, Duangmala K, et al. Molecular characterization of carbapenem-resistant Escherichia coli and
Acinetobacter baumannii in the Lao people's democratic republic. $J$ Antimicrob Chemother 2019;74:2810-21.

15 LOMWRU. Scientific Annual Report for 2018, 2018. Available: https://www.tropmedres.ac/_asset/file/lomwry-2018-annual-reportto-lao-moh.pdf [Accessed 09 Apr 2021].

16 Wyres KL, Nguyen TNT, Lam MMC, et al. Genomic surveillance for hypervirulence and multi-drug resistance in invasive Klebsiella pneumoniae from South and Southeast Asia. Genome Med 2020;12:11.

17 Stoesser N, Xayaheuang S, Vongsouvath M, et al. Colonization with Enterobacteriaceae producing ESBLs in children attending preschool childcare facilities in the Lao People's Democratic Republic. J Antimicrob Chemother 2015;70:1893-7.

18 Nakayama T, Ueda S, Huong BTM, et al. Wide dissemination of extended-spectrum $\beta$-lactamase-producing Escherichia coli in community residents in the Indochinese peninsula. Infect Drug Resist 2015;8:1-5.

19 Chang K. Epidemiology of extended spectrum beta-latamase in Lao PDR [Infectious disease fellowship]. Faculty of Postgraduate Studies, University of Health Sciences, Lao PDR, 2016.

20 Kantele A, Kuenzli E, Dunn SJ, et al. Dynamics of intestinal multidrug-resistant bacteria colonisation contracted by visitors to a high-endemic setting: a prospective, daily, real-time sampling study. Lancet Microbe 2021;2:e151-8.

21 Poupaud M, Putthana V, Patriarchi A, et al. Understanding the veterinary antibiotics supply chain to address antimicrobial resistance in Lao PDR: roles and interactions of involved stakeholders. Acta Trop 2021;220:105943.

22 Olaitan AO, Diene SM, Kempf M, et al. Worldwide emergence of colistin resistance in Klebsiella pneumoniae from healthy humans and patients in Lao PDR, Thailand, Israel, Nigeria and France owing to inactivation of the PhoP/PhoQ regulator mgrB: an epidemiological and molecular study. Int $J$ Antimicrob Agents 2014;44:500-7.

23 Hadjadj L, Baron SA, Olaitan AO, et al. Co-occurrence of variants of $m c r-3$ and $m c r-8$ genes in a Klebsiella pneumoniae isolate from Laos. Front Microbiol 2019;10:2720.

24 Iwanaga M, Sisavath LAY, Higa N, et al. Emergence of methicillin resistant Staphylococcus aureus in Laos. Jap. J. Trop. Med. Hyg. 1997;25:103-6.

25 Sithivong N, Phantouamath B, Chomlasak K, et al. Monitoring of drug resistant Staphylococcus aureus in People's Democratic Republic of Lao. Jap J Trop Med Hyg 2002;30:109-13.

26 Yeap AD, Woods K, Dance DAB, et al. Molecular epidemiology of Staphylococcus aureus skin and soft tissue infections in the Lao People's Democratic Republic. Am J Trop Med Hyg 2017;97:423-8.

27 Mayxay M, Castonguay-Vanier J, Chansamouth V, et al. Causes of non-malarial fever in Laos: a prospective study. Lancet Glob Health 2013;1:e46-54

28 Phetsouvanh R, Phongmany S, Soukaloun D, et al. Causes of community-acquired bacteremia and patterns of antimicrobial resistance in Vientiane, Laos. Am J Trop Med Hyg 2006;75:978-85.

29 Hongsakhone S. Fievre typhoid, etude epidemio - clinique et profil de sensibilite a l'hopital provincial d'Oudomxay, Laos - 4e promotion, 2002-2004 [MSc]. IFMT. IFMT, 2004.

30 Bounsavath N. Etiology of septicemia in Houaphan Provincal Hospital 2005-2008 [internal medicine]. Laos: University of Health Sciences: University of Health Sciences, 2008.

31 Chansamouth V, Thammasack S, Phetsouvanh R, et al. The aetiologies and impact of fever in pregnant inpatients in Vientiane, Laos. PLoS Negl Trop Dis 2016;10:e0004577.

32 Phongmany S, Phetsouvanh R, Sisouphone S, et al. A randomized comparison of oral chloramphenicol versus ofloxacin in the treatment of uncomplicated typhoid fever in Laos. Trans $R$ Soc Trop Med Hyg 2005;99:451-8.

33 Roberts T, Rattanavong S, Phommasone K, et al. Typhoid in Laos: an 18-year perspective. Am J Trop Med Hyg 2020;102:749.

34 Parry CM, Thieu NTV, Dolecek C, et al. Clinically and microbiologically derived azithromycin susceptibility breakpoints for Salmonella enterica serovars Typhi and Paratyphi A. Antimicrob Agents Chemother 2015;59:2756-64.

35 Le Thi Phuong T, Rattanavong S, Vongsouvath M, et al. Nontyphoidal salmonella serovars associated with invasive and noninvasive disease in the Lao People's Democratic Republic. Trans $R$ Soc Trop Med Hyg 2017;111:418-24.

36 Yamashiro T, Nakasone N, Higa N, et al. Etiological study of diarrheal patients in Vientiane, Lao People's Democratic Republic. J Clin Microbiol 1998;36:2195-9.

37 Darton TC, Thanh Tuyen H, Chung The H. Azithromycin resistance in Shigella spp. in Southeast Asia. Antimicrob agents chemother, 2018. 
38 Phantouamath B, Sithivong N, Phouthavanh T, et al. Drug susceptibility of Neisseria gonorrhoeae in Lao People's Democratic Republic. Jap J Trop Med Hyg 2003;31:117-20.

39 Thammalangsy S, Sihavong A, Phouthavane T, et al. The prevalence of lower genital tract infections among ante-natal care (ANC) clinic patients in two central hospitals, Vientiane, Lao People's Democratic Republic. Southeast Asian J Trop Med Public Health 2006;37:190-9.

40 Sihavong A, Phouthavane T, Lundborg CS, et al. Reproductive tract infections among women attending a gynecology outpatient department in Vientiane, Lao PDR. Sex Transm Dis 2007;34:791-5.

41 Phouangsouvanh S, Mayxay M, Keoluangkhot V, et al. Antimicrobial susceptibility of Neisseria gonorrhoeae isolates in Vientiane, Lao PDR. J Glob Antimicrob Resist 2018;13:91-3.

42 Moore CE, Sengduangphachanh A, Thaojaikong T, et al. Enhanced determination of Streptococcus pneumoniae serotypes associated with invasive disease in Laos by using a real-time polymerase chain reaction serotyping assay with cerebrospinal fluid. Am J Trop Med Hyg 2010;83:451-7.

43 Dubot-Pérès A, Mayxay M, Phetsouvanh R, et al. Management of central nervous system infections, Vientiane, Laos, 2003-2011. Emerg Infect Dis 2019;25:898-910.

44 lem V, Somphavong S, Buisson Y, et al. Resistance of Mycobacterium tuberculosis to antibiotics in Lao PDR: first multicentric study conducted in 3 hospitals. BMC Infect Dis 2013;13:275

45 Somphavong S, Berland J-L, Gauthier M, et al. First insights into the genetic characteristics and drug resistance of Mycobacterium tuberculosis population collected during the first national tuberculosis prevalence survey of Lao PDR (2010-2011). BMC Infect Dis 2019;19:851.

46 lem V, Dean A, Zignol M, et al. Low prevalence of MDR-TB in Lao PDR: results from the first national anti-tuberculosis drug resistance survey. Trop Med Int Health 2019;24:421-31.

47 Clarkson MC, Aguas R, Sweet K, et al. How many human pathogens are there in Laos? An estimate of national human pathogen diversity and analysis of historical trends. BMJ Glob Health 2020;5:e002972.

48 Dance DAB, Davong V, Soeng S, et al. Trimethoprim/ sulfamethoxazole resistance in Burkholderia pseudomallei. Int $J$ Antimicrob Agents 2014;44:368-9.

49 Boss J, Dance DAB, Chanthongthip A, et al. Antimicrobial susceptibility testing of Leptospira spp. in the Lao People's Democratic Republic using disk diffusion. Am J Trop Med Hyg 2019;100:1073-8.

50 Boonmar S, Markvichitr K, Chaunchom S, et al. Salmonella prevalence in slaughtered buffaloes and pigs and antimicrobial susceptibility of isolates in Vientiane, Lao People's Democratic Republic. J Vet Med Sci 2008;70:1345-8.

51 Boonmar S, Morita Y, Pulsrikarn C, et al. Salmonella prevalence in meat at retail markets in Pakse, Champasak Province, Laos, and antimicrobial susceptibility of isolates. J Glob Antimicrob Resist 2013;1:157-61.

52 Sinwat N, Angkittitrakul S, Coulson KF, et al. High prevalence and molecular characteristics of multidrug-resistant Salmonella in pigs, pork and humans in Thailand and Laos provinces. $J$ Med Microbiol 2016;65:1182-93.

53 Sunn K. Phenotypic and genotypic characteristics of extendedspectrum beta-lactamase (ESBL) production and colistin-resistance in Salmonella enterica and Escherichia coli isolated from pigs and their meat products in the border provinces between Thailand and Cambodia, Lao PRD and Myanmar [science in veterinary science and technology]. Chulalongkorn University, Thailand: Faculty of Veterinary Science, 2018.

54 Sychanh T, Chaunchom S, Pulsrikarn C. Salmonella prevalence in slaughtered buffaloes and cattle in Champasak Province, Lao People's Democratic Republic. Kasetsart J(Nat Sci) 2013;47:561-70.

55 Olaitan $\mathrm{AO}$, Thongmalayvong $\mathrm{B}$, Akkhavong $\mathrm{K}$, et al. Clonal transmission of a colistin-resistant Escherichia coli from domesticated pig to a human in Laos. J Antimicrob Chemother 2015;70:3402-4

56 Thu WP, Sinwat N, Bitrus AA, et al. Prevalence, antimicrobial resistance, virulence gene, and class 1 integrons of Enterococcus faecium and Enterococcus faecalis from pigs, pork and humans in Thai-Laos border provinces. $J$ Glob Antimicrob Resist 2019:18:130-8.

57 Quet F, Vlieghe E, Leyer C, et al. Antibiotic prescription behaviours in Lao People's Democratic Republic: a knowledge, attitude and practice survey. Bull World Health Organ 2015;93:219-27.

58 Keohavong B, Syhakhang L, Sengaloundeth S, et al. Rational use of drugs: prescribing and dispensing practices at public health facilities in Lao PDR. Pharmacoepidemiol Drug Saf 2006;15:344-7.
59 LOMWRU. Data from: hospital antimicrobial point prevalence survey 2017-2018. Vientiane, Laos: Lao-Oxford Mahosot Hospital Wellcome Trust Research Unit (LOMWRU), Mahosot Hospital, 2018.

60 Keohavong B, Vonglokham M, Phoummalaysith B, et al. Antibiotic prescription for under-fives with common cold or upper respiratory tract infection in Savannakhet Province, Lao PDR. Trop Med Health 2019;47:16

61 DHR. Lao adult antimicrobial prescribing guidelines. 1st edn. Department of Health Care and Rehabilitation (DHR) of Laos, 2020: 157.

62 DHR. Lao paediatric antimicrobial prescribing guidelines. 1st edn. Department of Health Car and Rehabilitation (DHR) of Laos, 2020: 155.

63 Haenssgen MJ, Charoenboon N, Xayavong T, et al. Precarity and clinical determinants of healthcare-seeking behaviour and antibiotic use in rural Laos and Thailand. BMJ Glob Health 2020;5:e003779.

64 Haenssgen MJ, Charoenboon N, Zanello G, et al. Antibiotic knowledge, attitudes and practices: new insights from crosssectional rural health behaviour surveys in low-income and middleincome south-east Asia. BMJ Open 2019;9:e028224.

65 Sihavong A, Lundborg CS, Syhakhang L, et al. Antimicrobial self medication for reproductive tract infections in two provinces in Lao People's Democratic Republic. Sex Transm Infect 2006;82:182-6.

66 Sihavong A, Lundborg CS, Syhakhang L, et al. Community perceptions and treatment-seeking behaviour regarding reproductive tract infections including sexually transmitted infections in Lao PDR: a qualitative study. J Biosoc Sci 2011;43:285-303.

67 Phonlavong C, Kitikannakorn N. General population's knowledge, beliefs, and care-seeking behavior about antibiotics: a crosssectional survey in Lao People's Democratic Republic. Thai J Pharm Prac 2019;10:411-20.

68 Khennavong M, Davone V, Vongsouvath M, et al. Urine antibiotic activity in patients presenting to hospitals in Laos: implications for worsening antibiotic resistance. Am J Trop Med Hyg 2011;85:295-302.

69 Syhakhang L, Freudenthal S, Tomson G, et al. Knowledge and perceptions of drug quality among drug sellers and consumers in Lao PDR. Health Policy Plan 2004;19:391-401.

70 Syhakhang L, Lundborg CS, Lindgren B, et al. The quality of drugs in private pharmacies in Lao PDR: a repeat study in 1997 and 1999. Pharm World Sci 2004;26:333-8.

71 Tabernero P, Swamidoss I, Mayxay M, et al. A random survey of the prevalence of falsified and substandard antibiotics in the Lao PDR. $J$ Antimicrob Chemother 2019;74:2417-25.

72 Theungphachan T. "Country report: Lao PDR." livestock \& fisheries products quality assurance unit, NAHC, DLF, 2012. Available: http:// webcache.googleusercontent.com/search? $\mathrm{q}=$ cache:http://lad.nafri. org.la/fulltext/3783-0.pdf [Accessed 09 Apr 2021].

73 Huynh TTT, Aarnink AJA, Drucker A. Pig production in Cambodia, Laos, Philippines, and Vietnam: a review. Asian J Agric Dev 2007;4:69-90.

74 Phanthavong S. Study on treatment of demodex canine disease of dogs [Bachelor's degree]. Lao National University, Faculty of Agriculture, 2016.

75 Innoula N. Study on the treatment of canine parvoviral enteritis by using antibiotic [Bachelor's degree]. Lao National University, Faculty of Agriculture, 2017.

76 Boudakham M. Study on the effectives of antibiotic for canine distemper [Bachelor's degree]. Lao National University, Faculty of Agriculture, 2017.

77 Keovilay P. Effectiveness of antibiotics on enteritis diseases in dogs [Bachelor's degree]. Lao National University, Faculty of Agriculture, 2017.

78 Labatut F, Suter I. The veterinary care of domesticated elephants in Laos by a mobile veterinary unit. GAJAH 2010;32:21-6.

79 Miyazato T, Tamaki Y, Sithivong N, et al. Antibiotic susceptibility and its genetic analysis of Vibrio cholerae non-01, non-0139 from environmental sources in Lao People's Democratic Republic. Trop Med Health 2004:32:245-8.

80 Caillet C, Sichanh C, Syhakhang L, et al. Population awareness of risks related to medicinal product use in Vientiane capital, Lao PDR: a cross-sectional study for public health improvement in low and middle income countries. BMC Public Health 2015;15:590.

81 WHO. Global antimicrobial surveillance system: manual for early implementation: World Health organization, 2015. Available: http:// apps.who.int/iris/bitstream/10665/188783/1/9789241549400_eng. pdf?ua $=1$ [Accessed 09 Apr 2021].

82 Cheah PY, Newton PN, Mayxay M. The first science café in Vientiane, Laos. Lancet 2016;388:1376. 
83 Vu TVD, Choisy M, Do TTN, et al. Antimicrobial susceptibility testing results from 13 hospitals in Viet Nam: VINARES 2016-2017. Antimicrob Resist Infect Control 2021;10:78.

$84 \operatorname{Lim} \mathrm{C}$, Takahashi E, Hongsuwan M, et al. Epidemiology and burden of multidrug-resistant bacterial infection in a developing country. Elife 2016;5:e18082.

85 Vlieghe ER, Phe T, De Smet B, et al. Bloodstream infection among adults in Phnom Penh, Cambodia: key pathogens and resistance patterns. PLoS One 2013;8:e59775.

86 Fund F. Laos. Available: https://www.flemingfund.org/countries/laos/ [Accessed 03 Jun 2021].
87 WHO. The launch of the antimicrobial resistance surveillance program in Lao People's Democratic Republic, 2018.

Available: https://www.who.int/laos/news/detail/11-07-2018 the-launch-of-the-antimicrobial-resistance-surveillanceprogram-in-lao-people-s-democratic-republic [Accessed 03 Jun 2021].

88 LOMWRU. Mahosot Microbiology Review (MMR). , 2013: 7, 1-29.

89 Elliott I, Phommasone K, Vongsouvath M. Staphylococcus aureus bacteraemia in the Lao People's Democratic Republic: antibiotic susceptibility patterns and clinical management. Lao Medical Journal 2012;3:3-15. 